\title{
Coherent synchrotron radiation and bunch stability in a compact storage ring
}

\author{
Marco Venturini* \\ Lawrence Berkeley National Laboratory, University of California, Berkeley, California, 94720, USA \\ Robert Warnock ${ }^{\dagger}$ and Ronald Ruth \\ Stanford Linear Accelerator Center, Stanford University, Stanford, California 94309, USA
}

James A. Ellison ${ }^{\S}$

Department of Mathematics and Statistics, University of New Mexico, Albuquerque, New Mexico, 87131, USA

(Received 26 March 2004; published 28 January 2005)

\begin{abstract}
We examine the effect of the collective force due to coherent synchrotron radiation (CSR) in an electron storage ring with small bending radius. In a computation based on time-domain integration of the nonlinear Vlasov equation, we find the threshold current for a longitudinal microwave instability induced by CSR alone. The model accounts for suppression of radiation at long wavelengths due to shielding by the vacuum chamber. In a calculation just above threshold, small ripples in the charge distribution build up over a fraction of a synchrotron period, but then die out to yield a relatively smooth but altered distribution with eventual oscillations in bunch length. The instability evolves from small noise on an initial smooth bunch of rms length much greater than the shielding cutoff.
\end{abstract}

DOI: 10.1103/PhysRevSTAB.8.014202

PACS numbers: 41.60.Ap, 29.27.Bd, 52.65.Ff

\section{INTRODUCTION}

The particles in a bunch traveling on a curved trajectory in free space emit synchrotron radiation with a broad spectrum of wavelengths. At wavelengths comparable to the bunch length or larger, the radiation from various particles is coherent, giving a radiated power proportional to $N^{2}$, where $N$ is the bunch population. Being so much larger than the incoherent power at shorter wavelengths, proportional to $N$, this coherent radiation could actually prevent operation of an electron storage ring if it were not suppressed by the effect of the metallic vacuum chamber surrounding the beam. This shielding effect was recognized in the 1940s, and was first computed in simple models of the vacuum chamber by Schwinger [1,2], Schiff [3], and Nodvick and Saxon [4].

In the model consisting of infinite parallel plates with separation $h$, with the particles moving on a circle of radius $R$ in the median plane, the coherent radiation is exponentially suppressed for wavelengths greater than about $\lambda_{0}$, where

$$
\lambda_{0}=2 h\left(\frac{h}{R}\right)^{1 / 2} .
$$

A cutoff of roughly the same value holds for more elaborate models $[5,6]$, for instance a smooth, resistive, toroidal chamber with rectangular cross section, if the transverse dimensions are of order $h$. This "shielding cutoff," which differs from the familiar waveguide cutoff $\lambda_{w}=2 h$ by the

\footnotetext{
*Electronic address: mventurini@lbl.gov

†Electronic address: warnock@slac.stanford.edu

${ }^{\ddagger}$ Electronic address: rruth@slac.stanford.edu

${ }^{\S}$ Electronic address: ellison@math.unm.edu
}

typically small factor $(h / R)^{1 / 2}$, is usually quite small compared to typical bunch lengths in storage rings. Coherent radiation of a certain wavelength $\lambda$ can be produced only if the Fourier spectrum of the bunch has an appreciable component of that wavelength. From this we might conclude that coherent synchrotron radiation (CSR) will not be a big effect in most storage rings, since if the bunch form is smooth and nearly Gaussian with rms spread $\sigma_{z}$, then the bunch spectrum cuts off quickly for $\lambda<2 \pi \sigma_{z}$. We must be cautious about this point, however, since there might be higher modes associated with some sort of small-scale structure on the bunch form. If at high current the field from coherent radiation is sufficiently strong to cause even more prominent small-scale structure, there might be an exponential buildup and eventual large-scale changes in the bunch form. A reason for worry in this regard is that the CSR wake field can be huge at wavelengths just smaller than the shielding cutoff. In the parallel plate model the maximum real part of $Z(n) / n$, where $Z$ is the longitudinal impedance, is given approximately in ohms by

$$
\left[\frac{\operatorname{Re} Z(n)}{n}\right]_{\max }=\frac{360}{e} \frac{h}{R} \approx 132 \frac{h}{R} \Omega .
$$

Here $n$ specifies an azimuthal mode of the electromagnetic field with wavelength $2 \pi R / n$. Following convention we write $Z(n)$ although it would be more appropriate to write $Z(n / R)$, thereby recognizing that $Z$ is a function of the wave number. For rings with small bending radii, like the example we shall study, the value (2) can be quite large compared to typical machine impedances, but those are usually most important at lower frequencies. Figure 1 shows $Z(n) / n$ for the parameters of the example treated presently. 


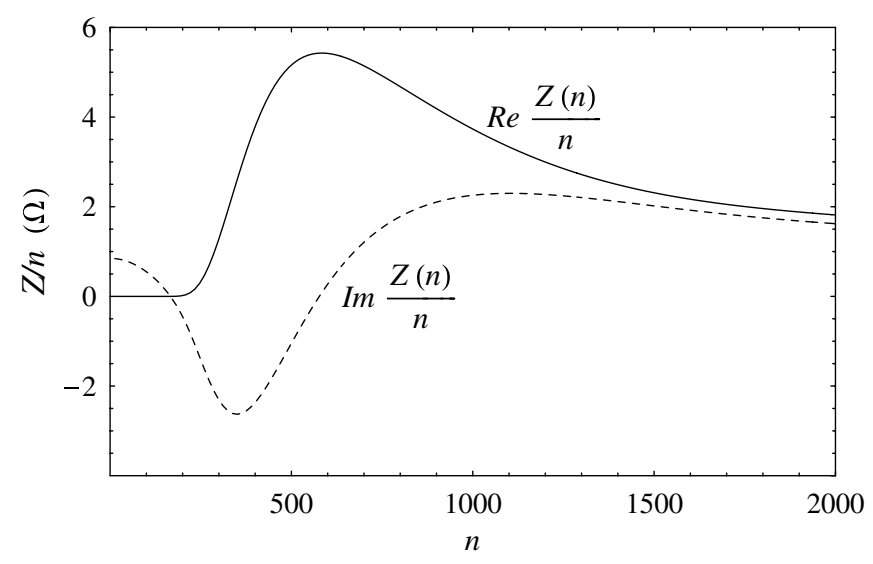

FIG. 1. Real (solid line) and imaginary (dashed line) part of $Z(n) / n$ for the parallel plate model with $h=1 \mathrm{~cm}, R=25 \mathrm{~cm}$, and energy $E_{0}=25 \mathrm{MeV}$.

The maximum value (2) occurs at $n \approx \pi \sqrt{2}(R / h)^{3 / 2}$, whereas the cutoff $\lambda_{0}$ corresponds to $n_{0}=\pi(R / h)^{3 / 2}$. [Note that this definition of the cutoff is somewhat arbitrary, being a point at which the impedance is still appreciable but falling rapidly as $n$ decreases. At $n=$ $\sqrt{2}(R / h)^{3 / 2}$ the ratio $\operatorname{Re} Z(n) / n$ is off by a factor of about 2000 from its maximum value.]

Although $\operatorname{Re} Z(n)$ is exponentially suppressed for wavelengths larger than the shielding cutoff, that is not true of $\operatorname{Im} Z(n)$. This is because we define the impedance to represent the entire longitudinal field for the chosen model of the vacuum chamber, not just the part due to curvature. At low energies there is a prominent space charge contribution to $\operatorname{Im} Z$, which in our example is large enough to cause noticeable potential well distortion with bunch shortening.

It is interesting to recall that the first observation of coherent synchrotron radiation [7] involved a nominal bunch length much larger than $\lambda_{0}$. Since the bunch came from a linac, which could easily produce a ragged, nonGaussian bunch profile, there was probably enough smallscale structure to allow coherent radiation in spite of shielding. Furthermore, the first evidence of CSR in existing storage rings [8-12] was under conditions with nominal bunch length greater than $\lambda_{0}$, but was associated with a microwave instability that could cause the bunch substructure necessary to overcome shielding [13]. Correspondingly, the observations showed only intermittent bursts of radiation. Now there is evidence of steady radiation at BESSY in a situation with a very short bunch achieved through a lattice with low momentum compaction [14].

In this paper we report a dynamical simulation that supports the picture of microbunching in a stored electron beam as described above. We attempt to understand the basic phenomenon in a model with a simplified picture of the collective force from CSR. The force is computed as though it came from a zero transverse emittance beam on a circular orbit between parallel plates, the radius of the orbit equated to the bending radius (not the average ring radius) of the actual machine. Aside from this representation of the force, we adopt the usual picture of longitudinal motion with the revolution time and slip factor for the actual ring.

For this first study we omit the usual wake fields due to vacuum chamber corrugations. These might play some role in a correct quantitative description of the instability leading to microbunching, as has been suggested in Refs. $[8,15]$. At least in some machines it seems that CSR alone may adequately account for the threshold of instability $[10,16]$.

Within the limitations of the model and discretization error in numerics, we find a threshold current at which microbunching evolves from small noise on a smooth bunch. Also, we follow characteristics of the bunch form up to a kind of saturation of the instability, in which smallscale structures die out to a large extent and oscillations of a relatively smooth but altered bunch form ensue.

This study was initiated to provide guidance in design of a compact storage ring [17], but we hope that the technical experience gained will also help in analysis of CSR experiments on existing storage rings $[8,9,11,12,14]$ and in design of a possible steady CSR source [16,18]. In particular, we have made progress in controlling a relatively new approach based on time-dependent Vlasov dynamics $[13,19]$, in a situation where close analysis of short wavelength phenomena is essential. Aspects of our technique may prove to be useful as well in the important problem of single-pass CSR in bunch compressors [20,21]. Currently there is much concern about microbunching in high energy bunch compressors with very small energy spread. This problem is being studied with macroparticle tracking, in which assessment of numerical noise tends to be difficult. A Vlasov description like that used here is possibly an interesting alternative. Maintaining a smooth distribution in phase space, it allows microbunching without the unphysical macroparticle phase space granularity.

In Sec. II we describe briefly the project that motivated this work. In Sec. III we review the single-particle equations of motion, and the Vlasov equation. Section IV gives the form of the collective force from shielded CSR that we assume. Section VA reviews the theory of the linearized Vlasov equation for a coasting beam in the frequency domain. The discussion follows Landau's original method, but we bring out mathematical details that are often overlooked, for instance the fact that one is dealing with an integral equation of the third kind. Section V B reports a first numerical exercise to test the Vlasov code, namely, a calculation of the instability threshold for a coasting beam. The result agrees well with the linear analysis. We also compute nonlinear evolution of the coasting beam. Our main numerical results, for the bunched beam, appear in Sec. VI. We treat both the advent of the instability and its "saturation" at longer times, emphasizing evolution of the Fourier spectrum of the bunch. Section VII contains a summary and the outlook for further work. 
Our computation of the collective force from CSR is done by means of the impedance rather than by using a closed expression for the wake potential as some authors prefer. A full discussion of the impedance formalism with parallel plate shielding is given in Ref. [22]. This includes questions of retardation that arise in the rigorous treatment of a deforming bunch, which cannot be treated by a wake potential. The practical application of the impedance requires a fast evaluation of a certain Fourier sum. In Appendix A we show how to relate this sum to the fast Fourier transform (FFT). Appendix B reviews the method used for time-dependent solution of the Vlasov equation.

\section{MOTIVATION: A COMPACT ELECTRON RING FOR COMPTON X-RAY PRODUCTION}

A compact electron storage ring has been proposed as a part of a Compton-scattering $x$-ray source [17]. The idea is to produce usable $\mathrm{x}$ rays from interaction of a fast recirculating bunch of electrons and a laser flash trapped in an optical cavity [23]. The size of the storage ring confining the electrons should be as small as possible in order to maximize the collision frequency. We consider an example with the following parameters:

$$
\begin{aligned}
E_{0} & =25 \mathrm{MeV}, \quad \sigma_{E} / E_{0}=3 \times 10^{-3}, \quad \sigma_{z}=1 \mathrm{~cm}, \\
\nu_{s} & =0.0184, \quad \omega_{s}=5.4 \mathrm{MHz}, \quad R=25 \mathrm{~cm}, \\
h & =1 \mathrm{~cm}, \quad N=6.25 \times 10^{9}=1 \mathrm{nC} .
\end{aligned}
$$

Here $E_{0}$ is the nominal energy, $\sigma_{E}$ and $\sigma_{z}$ are rms energy spread and bunch length, $\nu_{s}$ is the synchrotron tune, $\omega_{s}=$ $2 \pi f_{s}$ is the circular synchrotron frequency, $R$ is the bending radius of each of the four $90^{\circ}$ bends, $h$ is the vacuum chamber gap, and $N$ is the bunch population. The ring lattice consists of the combination of two double bend achromats joined by short drift sections to accommodate an rf cavity, injection devices, and an interaction section for a combined length of about $6.3 \mathrm{~m}$.

The small value of $R$ has raised concern that CSR could become a limiting factor because of the unfavorable scaling in this parameter. As indicated by Eqs. (1) and (2) a small radius of curvature makes the screening by the vacuum chamber less effective, causes the radiation fields to be more intense, and allows a larger portion of the bunch to radiate coherently. A first assessment of instability in the linear approximation can be carried out using Boussard's argument to replace the bunched beam stability problem by a roughly equivalent coasting beam problem. As is shown in Sec. VA, this leads to a current threshold for instability of $7.1 \mathrm{nC}$, about 7 times the intended design value. While this value may appear sufficiently safe, the question remains as to whether it would be possible, in view of eventual luminosity upgrades, to operate the machine above threshold. Moreover, one would like to corroborate the coasting beam analysis with a more realistic modeling of beam dynamics including the effect of bunching. This desire motivated the nonlinear, self-consistent calculation of beam dynamics presented in this paper.

An unusual feature of the proposed ring is that radiation damping and excitation due to incoherent synchrotron radiation play no significant role in beam dynamics. The damping time for a machine of this size and energy is of the order of $1 \mathrm{sec}$, much larger than the cycle time corresponding to the planned $100 \mathrm{~Hz}$ repetition rate. One cannot rely on possible beneficial effects of radiation damping to contain the emergence of instabilities and help relax the beam distribution above threshold. Instead, because of relatively small bunch sizes and low energy, intrabeam scattering is expected to be significant. Over a machine cycle $(10 \mathrm{~ms})$ both longitudinal and transverse emittances may double in size [24]. This growth time is still quite large, however, compared to the time for onset and saturation of the CSR instability, which amounts to a few synchrotron periods. Consequently, neglect of intrabeam scattering seems justified in the present study.

\section{EQUATIONS OF MOTION}

Our model of beam dynamics is based on the standard picture of longitudinal motion with linearized rf accelerating field, augmented with the longitudinal force from CSR. Transverse motion is neglected entirely.

The slip factor $\eta$ is defined as the constant relating a change in angular rotation frequency $\Omega_{r}$ to a change in momentum $P$, namely,

$$
\eta=-\frac{P_{0}}{\Omega_{0}}\left(\frac{d \Omega_{r}}{d P}\right)_{P_{0}}=\alpha-1 / \gamma_{0}^{2}
$$

Here $P_{0}$ and $\Omega_{0}$ are the nominal (design) values of momentum and revolution frequency, respectively, while $\alpha$ is the momentum compaction factor and $\gamma_{0}=E_{0} / m c^{2}$ is the Lorentz factor for the nominal energy. Note that some authors define $\eta$ with the opposite sign, and some call $\eta$ the momentum compaction.

As dynamical variables we choose the dimensionless coordinates

$$
q=\frac{z}{\sigma_{z}}, \quad p=-\operatorname{sgn}(\eta) \frac{E-E_{0}}{\sigma_{E}} .
$$

Here $z=s-s_{0}$ is the distance (in arc length along the reference trajectory) to the synchronous particle, being positive when the test particle leads. The deviation of energy from the nominal energy is $E-E_{0}$, and $\operatorname{sgn}(\eta)$ is 1 for $\eta>0$ and -1 for $\eta<0$. For the moment, $\sigma_{z}$ and $\sigma_{E}$ are regarded as arbitrary scale factors to render $q$ and $p$ dimensionless and of convenient magnitude. In these variables the standard linearized equations of motion [25] take the form

$$
\frac{d p}{d \tau}=-a q, \quad \frac{d q}{d \tau}=\frac{p}{a}, \quad \tau=\omega_{s} t,
$$


where $\omega_{s}$ is the angular synchrotron frequency and

$$
a=\frac{\beta_{0} \omega_{s} \sigma_{z}}{c} \frac{E_{0}}{|\eta| \sigma_{E}}, \quad \beta_{0}=v_{0} / c
$$

The system (6) has Hamiltonian

$$
H(q, p)=\frac{1}{2 a} p^{2}+\frac{a}{2} q^{2} .
$$

In a normal electron storage ring, equilibrated by radiation damping balancing quantum fluctuations, the phase space density function at low current would be

$$
f_{0}(q, p)=\exp (-H(q, p)) / 2 \pi .
$$

Recalling the definition of $p$ in (5), we see that if $\sigma_{E}$ is identified with the low current rms energy spread, then $a=$ 1 or

$$
\frac{\beta_{0} \omega_{s} \sigma_{z}}{c}=\frac{|\eta| \sigma_{E}}{E_{0}}
$$

where $\sigma_{z}$ is the low current rms bunch length. This is a well known formula, at least for $\beta_{0}=1$.

In our example we do not have normal equilibration, but we nevertheless choose $a=1$ as a matter of convenience in notation. Then if $\sigma_{z}$ is taken to be a nominal bunch length, Eq. (10) gives a definition of $\sigma_{E}$, which is now only a scaling constant to define the dimensionless variable $p$ of (5), not the rms energy spread.

If the beam current is sufficiently high, significant collective forces may arise. These include "geometric" wake forces generated from interaction of the beam with the surrounding environment. Our main interest here is in the additional collective force due to trajectory curvature, which entails both wake and precursor components. Whatever the collective force, it may in principle be computed from Maxwell's equations under boundary conditions at the chamber wall, given the charge and current densities defined by the phase space density of the beam. The exact phase space density, accounting for granularity of charge, is replaced in Vlasov theory by a smoothed density $f$, and the collective force is a functional of $f$.

In our one-dimensional model the Vlasov density is denoted by $f(q, p, \tau)$, and the collective force by $I_{c} F(q, f, \tau)$. We define $F$ to be positive when it contributes a positive term to $d E / d \theta$; i.e., it has the same sign as the collective electric field. The normalization of $F$ is chosen to give the current parameter $I_{c}$ the value

$$
I_{c}=\frac{\operatorname{sgn}(\eta) e^{2} N}{2 \pi \nu_{s} \sigma_{E}},
$$

where $N$ is the bunch population and $\nu_{s}$ is the synchrotron tune. In MKS units $I_{c}$ is in coulombs per volt. By (5) and (6) with $a=1$ the single-particle motion is governed by the equations

$$
\frac{d p}{d \tau}=-q-I_{c} F(q, f, \tau), \quad \frac{d q}{d \tau}=p
$$

The distribution function satisfies the Vlasov equation,

$$
\frac{\partial f}{\partial \tau}+p \frac{\partial f}{\partial q}-\frac{\partial f}{\partial p}\left[q+I_{c} F(q, f, \tau)\right]=0 .
$$

We normalize $f$ to have unit integral. Then the particle density on configuration space is

$$
\rho(q, \tau)=\int d p f(q, p, \tau)
$$

\section{COLLECTIVE FORCE DUE TO CSR}

We do not yet have a numerical method to compute CSR for arbitrary vacuum chamber walls and arbitrary particle orbits. If the vacuum chamber is modeled by parallel plates one can allow arbitrary orbits in planes parallel to the plates, enforcing field boundary conditions on the plates by the method of images. This would enable an account of transverse horizontal spread in the beam due to energy spread and angular deviation in horizontal motion. For this first exploration we adopt the parallel plate vacuum chamber, but for simplicity we assume that the horizontal spread does not have a major effect in the field produced by the beam. In fact, we compute the collective force as though it came from particles on a fictitious circular orbit with radius equal to the bending radius of the ring of interest. Thus we neglect not only transverse spread but also transient fields near magnet edges.

We work in cylindrical coordinates, with the $y$ axis perpendicular to the plates located at $y= \pm g, h=2 g$. We suppose that the charge/current distribution has the form of a "vertical ribbon beam." In the bunch frame the line density is $\lambda(\theta, t)$, and in the laboratory frame the normalized particle density $\rho$ and current density $\mathbf{J}$ are as follows:

$$
\begin{aligned}
\rho(r, \theta, y, t) & =\lambda\left(\theta-\omega_{0} t, t\right) \frac{\delta(r-R)}{R} H(y), \\
\mathbf{J} & =\left(J_{r}, J_{\theta}, J_{y}\right)=\left(0, Q \beta_{0} c \rho, 0\right), \\
\int_{0}^{2 \pi} \lambda(\theta, t) d \theta & =1, \quad \lambda(\theta+2 \pi, t)=\lambda(\theta, t), \\
\int_{-g}^{g} d y H(y) & =1,
\end{aligned}
$$

where $Q=\mp e N$ is the total charge and $\omega_{0}=\beta_{0} c / R$. Here $R$ is the bending radius of the ring, and the velocity $\beta_{0} c$ is identified with the nominal velocity of the ring. Note that $\omega_{0}$ differs from the revolution frequency of the ring, which is $\Omega_{0}=2 \pi \beta_{0} c / C$ for ring circumference $C$.

We shall compute the collective force as the mean value of the longitudinal electric field with respect to the transverse distribution: 


$$
\begin{aligned}
\mathcal{E}(\theta, t) & =\int_{0}^{\infty} r d r \int_{-g}^{g} d y \frac{\delta(r-R)}{R} H(y) E_{\theta}(r, \theta, y, t) \\
& =\int_{-g}^{g} E_{\theta}(R, \theta, y, t) H(y) d y .
\end{aligned}
$$

The corresponding induced voltage is $V(\theta, t)=$ $-2 \pi R \mathcal{E}(\theta, t)$. For the case of a rigid bunch the voltage is given in terms of the impedance function $Z(n, \omega)$ and the Fourier transform $\lambda_{n}$ of the line density $\lambda(\theta)$ as

$$
V(\theta, t)=\omega_{0} Q \sum_{n} e^{i n\left(\theta-\omega_{0} t\right)} Z\left(n, n \omega_{0}\right) \lambda_{n}
$$

In Ref. [22] we obtain the generalization of this formula for a deforming bunch, which involves the complete impedance $Z(n, \omega)$, not just its "diagonal" value $Z\left(n, n \omega_{0}\right)$. The latter, which we call the elementary impedance, corresponds to modes with phase velocity equal to the particle velocity. The exact form involves an integral over the frequency $\omega$ as well as a sum on $n$. In that paper we also find the first approximation to the exact formula and systematic corrections. The first approximation, which will be the basis for calculations reported here, amounts to replacing $\lambda_{n}$ in (17) by the time-dependent Fourier transform

$$
\lambda_{n}(t)=\frac{1}{2 \pi} \int_{0}^{2 \pi} d \theta e^{-i n \theta} \lambda(\theta, t) .
$$

Thus the voltage is computed as though the present charge distribution had existed for all time, meaning that retardation effects are not treated properly. Nevertheless, a first evaluation of corrections showed them to have a minor effect [26], at least over a limited time interval. We plan to make further checks in later work.

The impedance is given by [22]

$$
\begin{aligned}
\frac{Z(n, \omega)}{Z_{0}}= & \frac{(\pi R)^{2}}{\beta_{0} h} \sum_{p=1,3, \ldots} \Lambda_{p}\left[\frac{\omega \beta_{0}}{c} J_{n}^{\prime}\left(\gamma_{p} R\right) H_{n}^{(1) \prime}\left(\gamma_{p} R\right)\right. \\
& \left.+\left(\frac{\alpha_{p}}{\gamma_{p}}\right)^{2} \frac{n}{R} J_{n}\left(\gamma_{p} R\right) H_{n}^{(1)}\left(\gamma_{p} R\right)\right]
\end{aligned}
$$

Here $H_{n}^{(1)}=J_{n}+i Y_{n}$, where $J_{n}$ and $Y_{n}$ are Bessel functions of the first and second kinds, respectively, and $\alpha_{p}=$ $\pi p / h, \gamma_{p}^{2}=(\omega / c)^{2}-\alpha_{p}^{2}, \Lambda_{p}=2(\sin x / x)^{2}$, with $x=$ $\alpha_{p} \delta h / 2$. Here $h$ is the distance between the parallel plates, and $\Lambda_{p}$ is given for the case in which the vertical distribution $H(y)$ is constant for $|y| \leq \delta h / 2$ and zero otherwise. In MKS units $Z_{0}=120 \pi \Omega$. Evaluation of the Bessel functions requires appropriate asymptotic formulas for large $n$ [5]. Henceforth we follow convention in defining $Z(n)=$ $Z\left(n, n \omega_{0}\right)$.

Translating to the notation of the previous section, we have

$$
\begin{aligned}
F(q, f, \tau) & =-\frac{1}{Q} V\left(\frac{\omega_{0} \tau}{\omega_{s}}+\frac{\sigma_{z} q}{R}, \frac{\tau}{\omega_{s}}\right) \\
& =-\omega_{0} \sum_{n} \exp \left(\operatorname{in} \frac{\sigma_{z} q}{R}\right) Z(n) \lambda_{n}\left(\frac{\tau}{\omega_{s}}\right)
\end{aligned}
$$

where

$$
\lambda\left(\theta, \frac{\tau}{\omega_{s}}\right)=\frac{R}{\sigma_{z}} \int f\left(\frac{R}{\sigma_{z}} \theta, p, \tau\right) d p
$$

Note that (20) can be rewritten as a convolution of $\lambda$ with the Fourier transform of $Z(n)$, the latter being what is called the wake potential. Although one can give an approximate closed form for the wake potential [27], the computation of its convolution with $\lambda$ requires special care since it is sharply peaked in a region small compared to typical mesh cells in the Vlasov integration. Consequently, we prefer the impedance formulation, which also seems to provide an easier route to corrections to formula (20), as explained in [22].

Once we have adopted the approximation (20), so that the collective force depends only on $f$ evaluated at time $\tau$, we make the change of notation

$$
F(q, f, \tau) \rightarrow F(q, f(\cdot, \tau))
$$

The argument $f(\cdot, \tau)$ indicates that $F$ depends on the $f\left(q^{\prime}, p^{\prime}, \tau\right)$ at fixed $\tau$, with $\left(q^{\prime}, p^{\prime}\right)$ ranging over the whole phase space.

\section{CSR EFFECTS IN COASTING BEAMS}

\section{A. Linearized Vlasov equation}

In order to establish useful guidelines for our numerical study as well as a benchmark for the code, it is convenient to consider first the dynamics of coasting beams. The linear motion for coasting beams can be studied analytically and the results of the stability analysis extended to bunches - if the conditions for the validity of the Boussard criterion are met [28-30]. Boussard stated that a bunched beam and a coasting beam (with current equal to the peak current of the bunched beam) should exhibit similar thresholds for instability provided that the wavelength of the unstable mode is small compared to the bunch length. One can hope to apply this criterion to our case. Because of shielding CSR can only excite perturbations with wavelength $\lambda \lesssim \lambda_{0}=$ $2 h(h / R)^{1 / 2}$. For radius of curvature of the order of $R=$ $25 \mathrm{~cm}$ and chamber height $h=1 \mathrm{~cm}$ the cutoff is about $\lambda_{0}=4 \mathrm{~mm}$, smaller than a rms bunch length of $1 \mathrm{~cm}$ of interest here.

First, consider linearizing the Vlasov equation (13) around an equilibrium. Having set $f=f_{0}+f_{1}$, where $f_{1}$ is a small perturbation of equilibrium $f_{0}$, we find 


$$
\begin{aligned}
\frac{\partial f_{1}}{\partial \tau}+p \frac{\partial f_{1}}{\partial q}-\frac{\partial f_{1}}{\partial p}(q & +I_{c} F\left(q, f_{0}(\cdot, \tau)\right)- \\
& \frac{\partial f_{0}}{\partial p} I_{c} F\left(q, f_{1}(\cdot, \tau)\right)=0 .
\end{aligned}
$$

Next, in the spirit of Boussard's analysis we drop the rf focusing term, and consider a coasting beam with distribution function $f(q, p, \tau)=f_{0}+f_{1}$, periodic in $q$ with period $L=2 \pi R / \sigma_{z}$. Now coordinate space is the interval $[0, L]$, and the unperturbed distribution normalized to have unit integral is $f_{0}=\exp \left(-p^{2} / 2\right) /(\sqrt{2} \pi L)$. This distribution is indeed an equilibrium, since $F\left(q, f_{0}(\cdot, \tau)\right)=0$. A uniform charge distribution produces no wake force; equivalently, $Z(n)$ vanishes at $n=0$. The resulting linearized Vlasov equation can be used to investigate the linear dynamics of our original problem. Applying (20) and (21) we see that the equation of interest is

$$
\frac{\partial f_{1}}{\partial \tau}+p \frac{\partial f_{1}}{\partial q}+I_{d} \omega_{0} \frac{\partial f_{0}}{\partial p} \sum_{n=-\infty}^{\infty} Z(n) e^{i n q \sigma_{z} / R} \frac{R}{\sigma_{z}} \rho_{1 n}(\tau)=0
$$

where

$$
\rho_{1 n}(\tau)=\frac{1}{L} \int d q e^{-2 \pi n i q / L} \int d p f_{1}(q, p, \tau)
$$

with

$$
L=2 \pi R / \sigma_{z}, \quad I_{d}=L I_{c} / \sqrt{2 \pi} \sigma_{q} .
$$

The current parameter $I_{d}$ is chosen so that the charge density of the coasting beam is the same as the peak charge density of the bunched beam. We have assumed that the bunched beam is nearly Gaussian, so that its normalized particle density is approximately $\exp \left(-\left(q / \sigma_{q}\right)^{2} / 2\right) / \sqrt{2} \pi \sigma_{q}$. We seek solutions of (24) of period $L$ in $q$, and $L$ is typically so large that the periodicity imposes no substantial restriction on the form of the perturbation.

To find a solution we take the Fourier transform of (24) with respect to $q$ and the Laplace transform with respect to $t=\tau / \omega_{s}$. As in Eq. (7) of [22] we use the notation of Fourier analysis, taking $\omega$ to be the variable conjugate to time rather than the usual Laplace variable $s=-i \omega$. Nevertheless, our analysis is strictly in terms of the Laplace transform, since time integrals are on the positive real line and $\operatorname{Im} \omega>0$. The double transform of (24) exists if $\left|f_{1 n}(p, \tau)\right| \leq M e^{r_{n} t}$ for some positive $r_{n}$, and the series in (24) converges uniformly in $q$. We shall construct a solution of (24) assuming these conditions, and then observe that the solution in fact satisfies the same conditions. Taking the transform for $\operatorname{Im} \omega>r_{n}$ we have that

$$
\left[-i \frac{\omega}{\omega_{s}}+i n p \frac{\sigma_{z}}{R}\right] \hat{f}_{1 n}(p, \omega)+I_{d} \omega_{0} Z(n) \frac{R}{\sigma_{z}} f_{0}^{\prime}(p) \hat{\rho}_{1 n}(\omega)=\frac{1}{2 \pi \omega_{s}} f_{1 n}(p, 0)
$$

The initial value $f_{1 n}(p, 0)$ will be largely arbitrary, subject only to conditions of decay in $p$ and $n$, and smoothness in $p$. To be specific, let us assume convenient conditions (which can certainly be weakened); first, that $f_{1 n}(p, 0)$ is continuously differentiable in $p$, and that the function and its derivative decay at large $|p|$ as a power. We also need a condition on decay at large $n$, uniform in $p$, in order that the Fourier series converge. For simplicity, we may take $f_{1 n}(p, 0)=0,|n|>\bar{n}$.

Now (27) implies that the zero mode amplitude, $f_{10}(p, \tau)$, is actually independent of $\tau$, since its Laplace transform as a function of $\omega$ is a simple pole at $\omega=0$. Thus we can restrict attention to modes with $n \neq 0$, since only such modes are potentially unstable.

For $n \neq 0$ the transformed Eq. (27) is an integral equation for $\hat{f}_{1 n}(p, \omega)$ with separable kernel. With reference to $n$ and $\omega$ suppressed, it has the form

$$
d(p) \hat{f}_{1}(p)+a f_{0}^{\prime}(p) \int \hat{f}_{1}\left(p^{\prime}\right) d p^{\prime}=b f_{1}(p, 0)
$$

Clearly, any solution may be represented in its $p$ dependence as $\hat{f}_{1}(p)=\left(b f_{1}(p, 0)+\lambda f_{0}^{\prime}(p)\right) / d(p)$. Substituting this expression in (27) and solving for the constant $\lambda$, we find the solution of (27) as

$$
\begin{aligned}
\hat{f}_{1 n}(p, \omega)= & \frac{b_{n}}{d_{n}(p, \omega)}\left[f_{1 n}(p, 0)-a_{n} \frac{f_{0}^{\prime}(p)}{D_{n}(\omega)}\right. \\
& \left.\times \int \frac{f_{1 n}\left(p^{\prime}, 0\right) d p^{\prime}}{d_{n}\left(p^{\prime}, \omega\right)}\right],
\end{aligned}
$$

where

$$
\begin{array}{rlrl}
d_{n}(p, \omega) & =p-\frac{R \omega}{\sigma_{z} \omega_{s} n}, & a_{n}=I_{d} \omega_{0} \frac{Z(n)}{i n}\left(\frac{R}{\sigma_{z}}\right)^{2}, \\
b_{n}=\frac{R}{2 \pi i \omega_{s} \sigma_{z} n}, & D_{n}(\omega)=1+a_{n} \int \frac{f_{0}^{\prime}(p) d p}{d_{n}(p, \omega)} .
\end{array}
$$

By integrating (29) over $p$ we find the transform of the charge density as

$$
\hat{\rho}_{1 n}(\omega)=\frac{b_{n}}{D_{n}(\omega)} \int \frac{f_{1 n}(p, 0) d p}{d_{n}(p, \omega)}
$$

For sufficiently large $\operatorname{Im} \omega>0$ the dispersion function $D_{n}(\omega)$ has no zero, since it tends to 1 as $\operatorname{Im} \omega \rightarrow \infty$. Let $r_{n}$ be the largest non-negative number so that $D_{n}(\omega)$ has no zero for $\operatorname{Im} \omega>r_{n}$. Then (29) is analytic in $\omega$ for $\operatorname{Im} \omega>$ $r_{n}$. The inverse Laplace transform can be taken along a contour $\operatorname{Im} \omega=r>r_{n}$, and that contour can then be 
pushed down to the real axis, possibly encircling a pole from a zero of $D_{n}$ if $r_{n}>0$. [The limit of $\hat{f}_{1 n}(p, \omega)$ as $\omega$ approaches the real axis exists because of our assumption of smoothness of $f_{1 n}(p, 0)$ and $f_{0}^{\prime}(p)$ ]. The pole residue will have a factor $e^{r_{n} t}$, showing that the initial perturbation $f_{1 n}(p, 0)$ grows exponentially at a rate $1 / r_{n}$. Thus we justify, a posteriori, the assumption on large- $t$ growth that was made in deriving (27). For sufficiently small current $I_{c}$ we have $r_{n}=0$, and as the current is increased we expect to reach a value $I_{c}^{\text {th }}$ at which a zero of $D_{n}$ crosses the real axis to the upper half-plane, giving an instability in mode $n$. We also expect that one mode $n_{0}$, determined by specific properties of the impedance, will be the "most unstable," the first to destabilize with increasing current.

One might consider pushing the $\omega$ contour still farther, down to a semicircle at infinity in the lower half-plane, thinking to represent the complete distribution as a sum of pole contributions from all stable and unstable modes. This is problematic, however, and not of much practical interest. Analytic continuation to the lower plane of the integrals involving $f_{1 n}(p, 0)$ and $f_{0}^{\prime}(p)$ requires global analyticity of those functions in $p$. Smoothness of a distribution function seems a natural requirement, as an approximation to the actual granular particle distribution when the number of particles is large, but the much stronger requirement of global analyticity seems unmotivated. Even if an analytic function such as the Gaussian is chosen, the contribution of the semicircle at infinity need not be zero.

The Eq. (28) is a singular equation, classified as an integral equation of the third kind [31,32], owing to the zero of the factor $d_{n}(p)$ in its first term. The solution has a pole at that zero. In the linearized Vlasov equation for a bunched beam the analogous singularity must be handled less directly, since the solution of the integral equation is not known explicitly. In Ref. [33] it is shown that a simple change in the choice of unknown function is sufficient to remove the singularity and produce a scheme that is entirely analogous to the coasting beam theory.

To search for unstable modes we look for zeros of $D_{n}(\omega)$ in the upper half-plane, for the case of Gaussian $f_{0}$. In that case $D_{n}$ can be expressed in terms of the error function of complex argument $\quad w(z) \equiv e^{-z^{2}} \operatorname{erfc}(-i z) \equiv e^{-z^{2}}[1+$ $\left.2 i / \sqrt{\pi} \int_{0}^{z} \exp \left(\xi^{2}\right) d \xi\right]$, as defined in [34], Sec. 7.1.3. With the definition

$$
W(z)=\frac{1}{\sqrt{2 \pi}} \int \frac{p e^{-p^{2} / 2}}{p-z} d p=1+i z \sqrt{\frac{\pi}{2}} w(z / \sqrt{2}),
$$

the equation $D_{n}(\omega)=0$ reads

$$
\frac{I_{c} \omega_{0}}{\sqrt{2 \pi} \sigma_{q}}\left(\frac{R}{\sigma_{z}}\right)^{2} \frac{Z(n)}{n}=\frac{i}{W\left(\omega R /\left(\omega_{s} \sigma_{z} n\right)\right)},
$$

where $I_{c}$ is defined in (11). Remember that $E_{0}, \beta_{0}, \sigma_{E}, \sigma_{z}, \nu_{s}, \omega_{s}, N$ are design parameters for the ring of interest. On the other hand, $R$ is the bending radius rather than the average radius of the ring, and the imped- ance is computed as though it came from a bunch on a circular path of radius $R$ with angular revolution frequency $\omega_{0}=\beta_{0} c / R$.

Henceforth we report results for the parameters of Eqs. (3), suitable for the compact ring discussed in Sec. II. The energy is assumed to be above transition, so that $\operatorname{sgn}(\eta)=1$ in (11). We suppose that the lattice is designed so that the slip factor $\eta$ satisfies (10) when the other parameters in that equation are from (3). In Sec. VI A we find the value 0.961 for the normalized equilibrium bunch length $\sigma_{q}$. For the present approximate calculation we put $\sigma_{q}=1$.

A convenient way to represent mode stability as predicted by (33) is by means of a Keil-Schnell diagram; see Fig. 2. In the complex plane one draws a stability boundary (dashed line in the picture) defined parametrically by the real and imaginary components of $-i / W(\Omega)$ for $\operatorname{Im} \Omega=0$ as we let $\operatorname{Re} \Omega$ run from $-\infty$ to $+\infty$. In the same plane we can also locate the negative of the left-hand side (LHS) of Eq. (33) as a function of $n$ for specified values of the current (solid lines in the picture). Points falling outside the Keil-Schnell curve identify unstable modes with positive imaginary part of frequency $\operatorname{Im} \omega>0$. Those falling inside represent stable modes. In Fig. 2 the thick line corresponds to a normalized current $I_{c}=0.8183 \mathrm{pC} / \mathrm{V}$. The fact that the thick curve in the picture is tangent to the stability boundary qualifies $I_{c}=0.8183 \mathrm{pC} / \mathrm{V} \quad(Q=$ $7.1 \mathrm{nC})$ as the current threshold. The most unstable mode has mode number $n=702$ (marked in the picture) corresponding to a wavelength $\lambda=2 \pi R / n=2.2 \mathrm{~mm}$.

The so-called "Keil-Schnell criterion" is an approximation to (33) obtained by putting $W=1$ and replacing $Z(n) / n$ by the maximum value of $|Z(n) / n|$. The threshold obtained from the exact equation is about $20 \%$ larger than the prediction of the Keil-Schnell criterion.

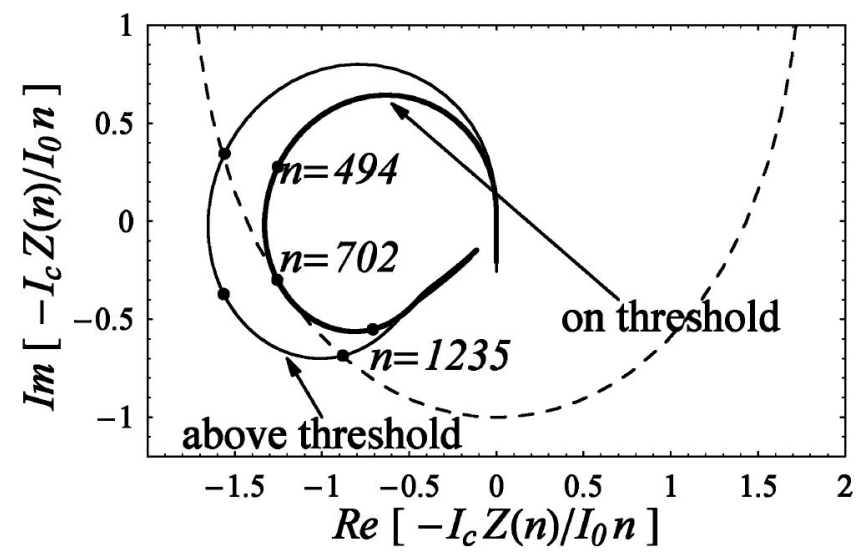

FIG. 2. Keil-Schnell stability diagram. The dashed line defines the onion-shaped stability boundary characteristic of an unperturbed distribution that is Gaussian in momentum. The solid lines represent $-I_{c} Z(n) / I_{0} n$ with $I_{c}$ equal to the critical value $I_{c}^{\text {th }}=0.8183(Q=7.1 \mathrm{nC})$ for instability (thicker line) and $I_{c}=$ 1.018 (thinner line). $I_{0}=\sqrt{2 \pi} \sigma_{q}\left(\sigma_{z} / R\right)^{2} / \omega_{0}$. 


\section{B. Numerical solution of the nonlinear Vlasov equation}

For numerical integration of the Vlasov equation we define the distribution function $f$ in terms of its values on a $(2 \mathcal{N}+1) \times(2 \mathcal{N}+1)$ grid with both (normalized) position and momentum $q$ and $p$ belonging to the interval $[-\kappa, \kappa]$ with $\kappa \simeq 6.04$. We used the method of Appendix A to compute the collective force, and the method of Appendix B to integrate the Vlasov equation. In the case of the coasting beam, the distribution function is constructed so that it is periodic in $q$ with period $2 \kappa$; i.e., $f(-\kappa, p)=f(\kappa, p)$ is imposed in the interpolation scheme described in Appendix A. This period is much smaller than the period $L=2 \pi R / \sigma_{z}$ of our basic Fourier analysis, but still much larger than the wavelengths of the unstable modes that we study. It would be impractical and unnecessary to use period $L$ in numerical work. We use the same $\kappa$ for both the bunched and coasting cases. Since the number of mesh points per unit distance is then the same, we can expect to resolve ripples of similar size in either case.

To check the performance of the Vlasov solver we first calculated the current threshold and compared the outcome with the linear theory. We started by placing a small sinusoidal perturbation in space on top of a distribution that is Gaussian in momentum and uniform in space:

$$
f=\frac{e^{-p^{2} / 2}}{\sqrt{2 \pi}}\left[1+A \sin \left(\frac{n q \sigma_{z}}{R}\right)\right]
$$

with $n=702$ corresponding to a wavelength $\lambda=$ $2 \pi R / n=2.2 \mathrm{~mm}$ (see Sec. VA).

Above threshold and after a short transient the charge density perturbation is well approximated as a traveling wave with exponentially growing amplitude $\propto$ $e^{\nu_{I} \tau} \sin \left(n q \sigma_{z} / R-\nu_{R} \tau\right)$, where $\nu_{R}$ and $\nu_{I}$ are the real and imaginary part of $\nu=\omega / \omega_{s}$ with $\omega$ given by $D_{n}(\omega)=0$. This can be seen by taking the inverse Laplace transform of (31). Pushing the $\omega$ contour to the real axis, we get the traveling wave from the pole contribution (multiplied by $e^{i n \theta}$ ), plus a background from the integral along the real axis. The latter can be understood as the source of the transient. We set the initial value of the perturbation amplitude to be small enough to avoid nonlinearities, $A=10^{-3}$, and computed the amplitude of the mode versus time up to $\tau=0.6$ for different values of the current parameter $I_{c}$. A logarithmic plot of the mode amplitude is reported in Fig. 3. The growth rates are computed by numerical fitting (upon discarding the initial transient). The resulting dependence of the growth rates on $I_{c}$ was then used to estimate the threshold by interpolation.

To check convergence to the theoretical value $I_{c}^{\text {th }}=$ $0.8183 \mathrm{pC} / \mathrm{V}$, we repeated the calculation for various choices of the grid sizes $\mathcal{N}=200,400$, and 600 and found $I_{c}^{\text {th }}=0.8341,0.8202,0.8189 \mathrm{pC} / \mathrm{V}$, respectively. Coarser grids appear to overestimate the thresholds. The smallest wavelengths resolved by these three grids (twice
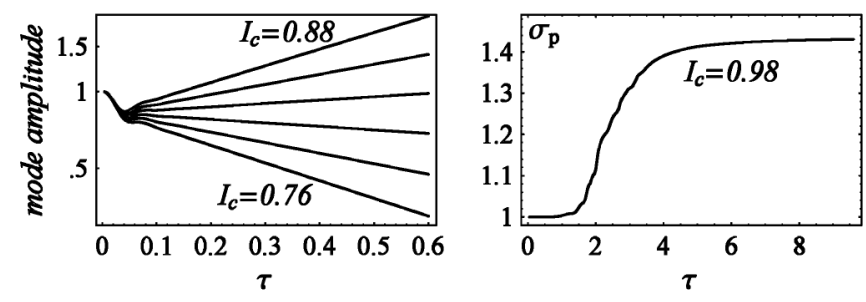

FIG. 3. Coasting beam. Left: Logarithm of the amplitude of Fourier mode $(n=702)$ versus time for six values of the current parameter $I_{c}$ (between $I_{c}=0.76$ and $I_{c}=0.88$ in steps of 0.024). Right: Evolution of normalized rms energy spread $\sigma_{p}$. In both pictures $\tau$ is the normalized time.

the size $\kappa \sigma_{z} / \mathcal{N}$ of a cell in the grid) are respectively $0.6,0.3,0.2 \mathrm{~mm}$ (to be compared with the perturbation wavelength $\lambda=2.2 \mathrm{~mm}$ ). Charge conservation during the calculation, which is one figure of merit to evaluate the overall accuracy, was about one part in $10^{5}$ or better, and improved with the density of mesh points.

Having gained confidence in the code we proceeded to follow the evolution of the instability over a longer time into the nonlinear regime. The results reported in Fig. 4 [which were obtained with $I_{c}=0.98$ and an initial perturbation as in (34) with $n=702$ ], represent some typical behavior of beam dynamics for a wide range of currents above threshold. The perturbation undergoes an initial exponential growth, then reaches saturation during the advent of a richer mode spectrum, eventually relaxing to some sort of pseudostationary distribution. Saturation of the energy spread is seen in the graph on the right in Fig. 3 and also in the third line of Fig. 4.

The resonance which in Fig. 4 is already apparent at time $\tau=1.2$ can be interpreted in terms of particle-wave interaction. When a single unstable mode dominates, the coherent force in (24) is proportional to $e^{\nu_{I} \tau} \sin \left(n q \sigma_{z} / R-\right.$ $\left.\nu_{R} \tau\right)$, so that the single-particle motion will look like pendulum motion in a comoving frame, over a restricted time interval in which the variation of $e^{\nu_{I} \tau}$ is not too severe. Particles with momentum near the perturbation phase velocity $p=\nu_{R} R / \sigma_{z} n$ undergo "resonant trapping" and cause the appearance of a "knee" on the profile of the momentum distribution (with size comparable to the width of the resonance). In the framework of a quasilinear theory this phenomenon sets the stage for the onset of saturation. As time progresses one observes a widening and distortion of the resonance islands and the appearance of "tongues" in the phase space distribution, branching outward and resulting in an enlargement of momentum spread. At a later time the charge density becomes smoother and the momentum distribution settles to some profile persistent in time as large-scale structures in phase space appear to get washed away. This process is reflected by the evolution of the Fourier spectrum of the charge density; see the bottom row in Fig. 4. The initial spectrum is a delta function at $m=702 / 13=54$. The nonlinearities first generate a cas- 

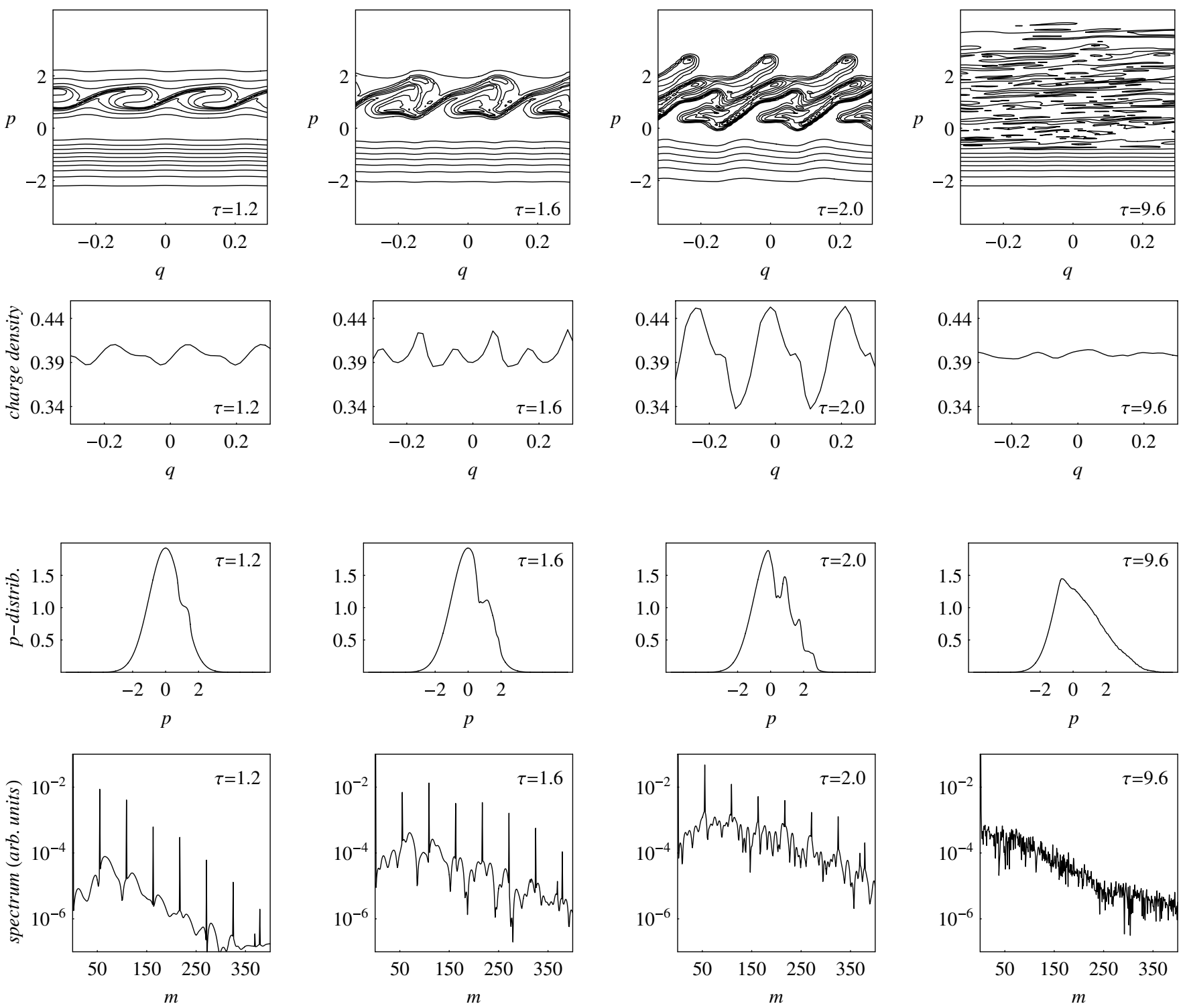

FIG. 4. Evolution in time of coasting beam by effect of CSR. Instability initiated by a small perturbation with mode number $n=702$ (wavelength $\lambda=2.2 \mathrm{~mm}$ ). From top to bottom: snapshots of contour plots of phase space density (top row), charge density (second row), momentum distribution (third row), and spectrum of charge density (bottom row). Pictures are taken at (normalized) time $\tau=$ 1.2, 1.6, 2.0, and 9.6. In the bottom row the abscissa is $m=n / 13$, where $n$ is the mode number (see text). The plots in the top two rows show only a small part of the full grid in $q$.

cade of modes with mode numbers that are multiples of 702 and eventually a smoother spectrum. While some of the smoothing may be an artifact of the numerical integration, the qualitative behavior appears to be independent of the choice of mesh and time step, suggesting that we have believable representations of the exact solution.

There are a couple of conclusions that we can draw at this point. The first is that our numerical modeling of the coasting beam correctly displays the onset of the instability in accordance with the linear theory and that this instability leads to the appearance of a corrugation in the charge density (microbunching). The second conclusion is that a mechanism for the removal of the microbunching is provided by the intrinsic nonlinearities of the system.

\section{CSR EFFECTS IN BUNCHED BEAMS}

\section{A. Equilibrium distribution}

Because of the relatively low energy of the beams we are considering, the space charge component of the impedance results in a small but noticeable potential well distortion. Since we do not want the stability analysis to be affected by a mismatch, we consider initial distributions that are stationary solutions of the Vlasov equation, thus accounting for the potential well distortion. For convenience of calculation we chose those stationary solutions to be Haïssinski distributions $f_{0}$. These have the form $f_{0}=e^{-p^{2} / 2} \rho_{0}(q) / \sqrt{2} \pi$, where $\rho_{0}(q)$ satisfies the equation 


$$
\rho_{0}^{\prime}(q)=\left(-q-I_{c} F\left(q, f_{0}(\cdot)\right)\right) \rho_{0}(q),
$$

with normalization $\int \rho_{0}(q) d q=1$. Because we expect that an equilibrium distribution will be relatively smooth, only the low-frequency part of the impedance should be relevant in shaping its profile. The Fourier spectrum of a smooth distribution representing a bunch of length $\sigma_{z}$ traveling along a circular orbit of radius $R$ is significant only for mode numbers smaller than a small multiple of the ratio $R / \sigma_{z}$. For the choice of parameters (3) relevant for the present study this number is well below the shielding cutoff for the real part of the impedance; see Fig. 1. Moreover, because the imaginary part of $Z(n) / n$ is nearly constant over the bunch spectrum, we conclude that the effective impedance shaping the Haïssinski solution is purely capacitive. In other words, for the purpose of determining the equilibrium distribution the collective force can be modeled as being proportional to the space derivative of the bunch distribution. This can be seen easily. Having defined $\hat{Z} \equiv \lim _{n \rightarrow 0} \operatorname{Im}(Z(n) / n)$ we have

$$
\begin{aligned}
F(q, \tau) & \simeq i \omega_{0} \sum_{n=-\infty}^{\infty} \operatorname{Im}\left[Z_{n}\left(n \omega_{0}\right)\right] e^{i n q \sigma_{z} / R} \lambda_{n} \\
& \simeq \omega_{0} \frac{R}{\sigma_{z}} \frac{\partial}{\partial q} \sum_{n=-\infty}^{\infty} \hat{Z} e^{i n q \sigma_{z} / R} \lambda_{n} \\
& =\omega_{0} \hat{Z}\left(\frac{R}{\sigma_{z}}\right)^{2} \frac{d}{d q} \rho_{0}(q) .
\end{aligned}
$$

Under the assumption that the potential well distortion is not too large we can estimate the relative variation of rms bunch length of the Haïssinski solution as

$$
\Delta\left\langle q^{2}\right\rangle=-\frac{1}{4 \sqrt{\pi}}\left(\frac{R}{\sigma_{z}}\right)^{2} \omega_{0} I_{c} \hat{Z} .
$$

Because $\hat{Z}>0$, the effect of the space charge part of the impedance is to shorten the bunch. An example of Haïssinski profile is shown in Fig. 5. For this plot the value of the current parameter $I_{c}=0.844 \mathrm{pC} / \mathrm{V}$ is close to the threshold for instability.

\section{B. Numerical solution of the nonlinear Vlasov equation}

Our first task is to determine the current threshold for instability. We superimposed a sinusoidal perturbation to the charge density with mode number corresponding to the most unstable mode expected by the linear coasting beam theory. Because of rf focusing past the initial transient, the growth of the modes is not purely exponential, as can be observed from Fig. 6. Somewhat arbitrarily we define growth rate in terms of an exponential fit of points falling in the interval $\tau \in[0.3,0.6]$. By doing so we find an estimate for the critical current $I_{c}=0.836 \mathrm{pC} / \mathrm{V}$ (obtained with a $800 \times 800$ mesh), or $7.20 \mathrm{nC}$ of charge. This should be compared with the critical value we obtained by applying the Boussard criterion, $I_{c}=$

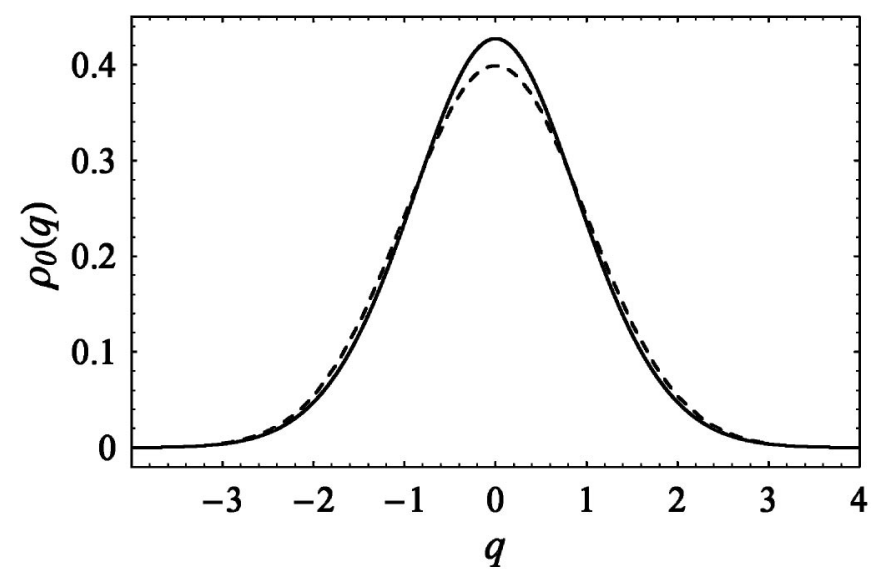

FIG. 5. Charge density of a Haïssinski solution (solid line) for $I_{c}=0.844 \mathrm{pC} / \mathrm{V}(h=1 \mathrm{~cm}$ and $R=25 \mathrm{~cm})$. The normalized rms bunch length is $\sigma_{q}=0.9609$. The dashed line represents a Gaussian bunch with unity rms length. The impedance yields the complete longitudinal field, so that it contains a large space charge component at low frequencies when the beam has low energy.

$0.8183 \mathrm{pC} / \mathrm{V}$. The latter number is reduced to 0.786 if we include the factor $1 / \sigma_{q}$, previously set equal to 1 . We conclude that the Boussard criterion is consistent with our findings within about $6 \%$. As in the calculation of coasting beams we found little variation in the estimate of the critical current as we increased the grid size to $1201 \times$ 1201 (in which case $I_{c}^{\text {th }}=0.833 \mathrm{pC} / \mathrm{V}$ ), while a coarser $401 \times 401$ grid resulted in a value $I_{c}^{\text {th }}=0.855 \mathrm{pC} / \mathrm{V}$, larger by a few percent.

Over a longer time the behavior of the bunch and that of a coasting beam differ in some regards. However, they share the feature that the nonlinearities cause a relaxation of much of the short scale density perturbation that is seen after onset of the instability. This process develops quickly and takes place within one or two synchrotron periods.

The instability originates in the center of the bunch where the charge density is the largest. A microbunch structure emerges (see Fig. 7) in the form of a ripple on the density profile with current-dependent amplitude. As CSR is emitted forward it mostly affects the front of the
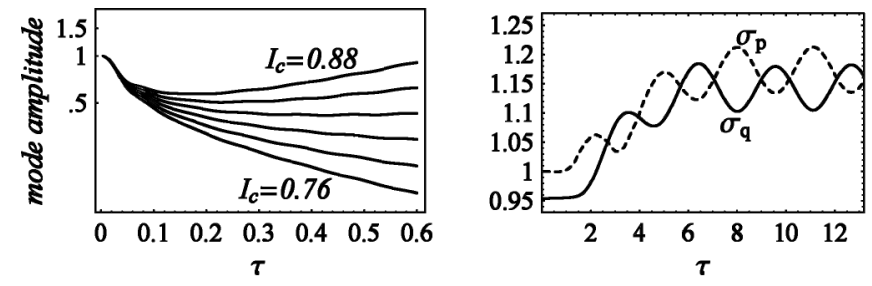

FIG. 6. Bunched beam. Left: Logarithm of the amplitude of Fourier mode $n=702$ versus time for six values of the current parameter $I_{c}$ (between $I_{c}=0.76$ and $I_{c}=0.88$ in steps of 0.024). Right: normalized rms bunch length $\sigma_{q}$ (solid line) and energy spread $\sigma_{p}$ (dashed line) versus time for $I_{c}=0.98$. 

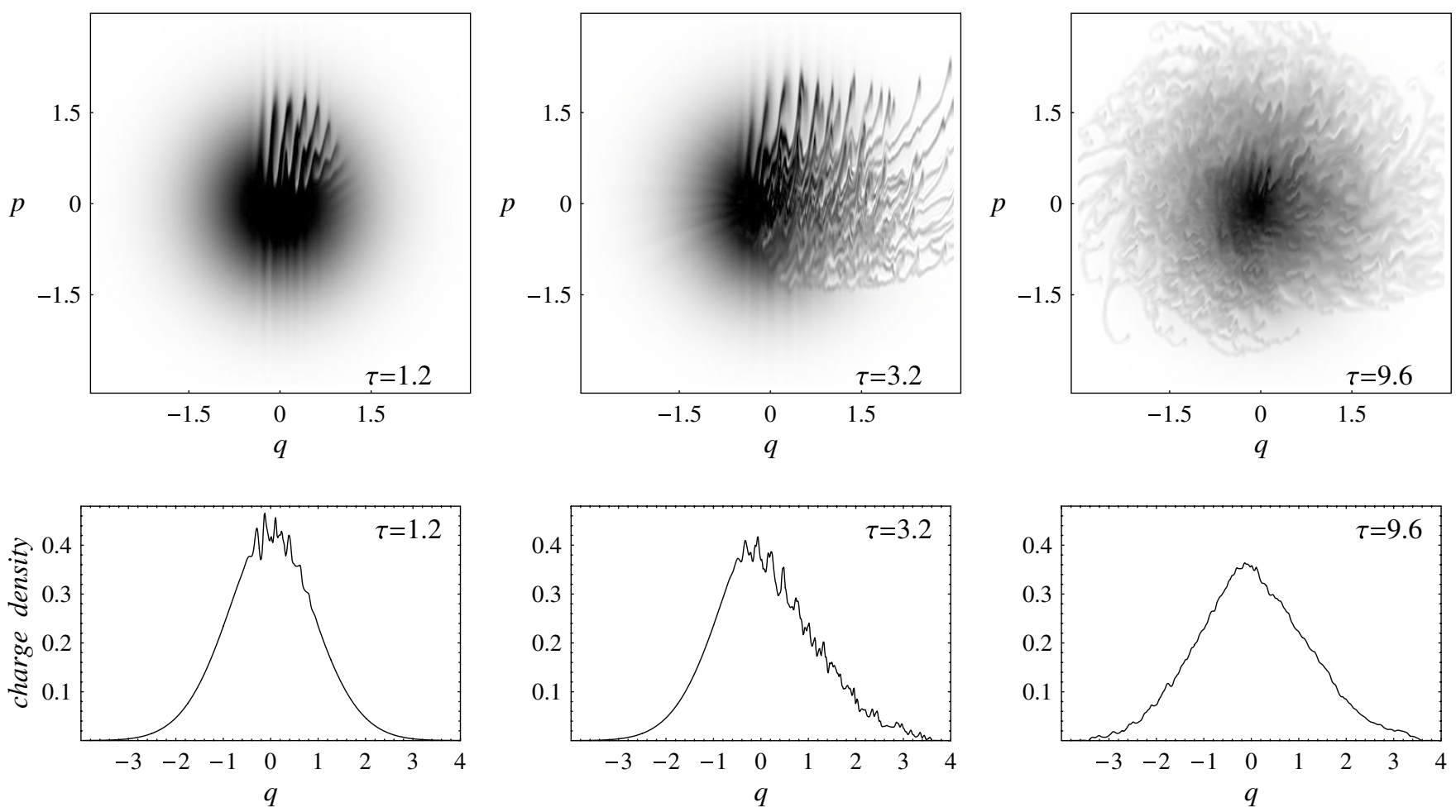

FIG. 7. Time evolution of bunch under effect of CSR. Density plots in phase space (top row) and charge density (second row). Pictures are taken at (normalized) time $\tau=1.2,3.2$, and 9.6. Instability initiated by a small perturbation with mode number $n=702$ (wavelength $\lambda=2.2 \mathrm{~mm}$ ). A unit of $q$ corresponds to $1 \mathrm{~cm}$.

bunch and causes the appearance of filaments in the bunch density in phase space (see Fig. 7 at $\tau=3.2$ ). This resulting perturbation in the bunch distribution first appearing in the head of the bunch is then carried over to the back and then around by the rotation in phase space from $\mathrm{rf}$ focusing. Because no additional relaxation forces (such as radiation damping) are in action, this imbalance results in a persistent quadrupolelike motion that can clearly be observed in the evolution of the rms bunch length and energy spread (see Fig. 6). After about two synchrotron periods the bunch distribution in phase appears to freeze as a larger momentum spread and smaller bunch density move the bunch away from the unstable condition. The quadrupole pulsation continues indefinitely. In calculations not shown here we have followed the evolution over hundreds of synchrotron periods and noticed very little change over time.

\section{SUMMARY AND OUTLOOK}

Coherent synchrotron radiation is currently attracting much attention both as a mechanism for generating usable radiation and as a source of potentially harmful instabilities. In this paper we focused on the dynamical effects of CSR and reported on our attempts to develop a simplified but hopefully still sufficiently accurate model for studying instabilities. We investigated the longitudinal beam dynamics by looking for numerical solutions of a Vlasov equation in 1 degree of freedom with attention paid to the specific case of a compact storage ring. However, aspects of the results we obtained are believed to apply to more conventional storage rings as well.

The main result of our numerical investigation could be summarized in the finding of (i) a current threshold for instability very close to the value predicted by linear theory for coasting beams (confirming the validity of Boussard's criterion to an accuracy of 6\%), (ii) emergence of "microbunching" (i.e., a charge density modulation on the beam density profile) above threshold as a signature of the instability, and (iii) rapid saturation (within two synchrotron periods) of the instability and smoothing of the bunch density.

In a previous paper [13] the creation and smoothing of microbunching was related to the appearance of bursts of coherent radiation as recently detected in several light sources. That was for cases in which radiation damping acts as a relaxation mechanism restoring the conditions for instability and causing a recurrence of bursting. No such mechanism is in place in the compact ring of interest in this paper, where above threshold a CSR driven instability would result in a persistent (and possibly unacceptable) emittance degradation. It is a fact worth emphasizing that the smoothing of the microbunching, as demonstrated by our calculation, occurs without operation of dissipative forces.

Our current numerical model does not account fully for retardation effects, since at each time step we approximate 
the collective force by a formula that uses only the present value of the charge density. In Ref. [22] we developed corrections to this picture which can be included at relatively low computational cost. A first evaluation of the corrections showed them to be of minor importance on a limited time interval [26], but computations for longer times remain to be done. Other investigations are on the agenda for further work: repeating the calculations with an alternative model of the vacuum chamber [5], evaluating the effects of horizontal transverse spread in the beam, and the effects of noncircular orbits.

Our solutions of the nonlinear Vlasov equation were computed by the method of local characteristics, also called the semi-Lagrangian or discretized PerronFrobenius (PF) method. The considerable value of this method for beam dynamics has been recognized only recently, and much work remains to be done in exploring its various implementations and extensions to higher dimensional phase space. We have not evaluated alternative techniques for the present problem, for instance the macroparticle approach [35] or the nonlinear $\delta f$ method [36]. In a tentative judgment we prefer our method for offering lower noise than the macroparticle technique, and for being more appropriate than $\delta f$ for dynamics far from equilibrium.

\section{ACKNOWLEDGMENTS}

Part of the work of M. V. was carried out at SLAC. Our research was supported in part by Department of Energy Contracts No. DE-AC03-76SF00515, No. DE-AC0376SF00098, and No. DE-FG03-99ER41104.

\section{APPENDIX A: NUMERICAL TREATMENT OF FOURIER TRANSFORMS}

For the Vlasov integration we need a numerical approximation to the Fourier transform (18). Suppressing time dependence and noting (21) and (14), we have

$$
\begin{aligned}
\lambda_{n} & =\frac{1}{2 \pi} \int_{-\pi R / \sigma_{z}}^{\pi R / \sigma_{z}} e^{-i n q \sigma_{z} / R} \rho(q) d q \\
& =\frac{1}{2 \pi} \int_{-\kappa}^{\kappa} e^{-i n q \sigma_{z} / R} \rho(q) d q .
\end{aligned}
$$

Here $\kappa$ defines the boundary of the $q$ mesh, $\rho(q)$ being zero by definition outside the interval $[-\kappa, \kappa]$. Typically $\kappa$ is around six or seven in the bunched beam case, much smaller than $\pi R / \sigma_{z}$. We take a uniform $q$ mesh consisting of the points

$$
q_{j}=j \kappa / \mathcal{N}-\kappa, \quad j=0,1, \ldots, 2 \mathcal{N},
$$

where a typical value of $\mathcal{N}$ is 200-600. Since we have to compute the transform at every time step, it is important to save time by employing the FFT. Some tricks are required to get the results we need from the FFT.
Recall that the FFT supplies the sum

$$
\frac{1}{J} \sum_{j=0}^{J-1} e^{-2 \pi i m j / J} f\left(\theta_{j}\right), \quad m=0,1, \ldots, J-1,
$$

which is the result of applying the trapezoidal rule to approximate the transform of a periodic $f(\theta)$,

$f_{m}=\frac{1}{2 \pi} \int_{0}^{2 \pi} e^{-i m \theta} f(\theta) d \theta, \quad d \theta=2 \pi / J, \quad \theta_{j}=2 \pi j / J$.

Correspondingly, let us evaluate (A1) by the trapezoidal rule, using values of $\rho$ on the mesh (A2). We find

$$
\lambda_{n}=\frac{\kappa e^{i \pi \mu}}{2 \pi \mathcal{N}} \sum_{j=0}^{2 \mathcal{N}-1} e^{-2 \pi i \mu j /(2 \mathcal{N})} \rho\left(q_{j}\right), \quad \mu=\frac{n \sigma_{z} \kappa}{\pi R}
$$

To put this sum in the standard form of an FFT, we adjust $\kappa$ (from whatever value we first assumed) to make $\Delta n$ an integer, where

$$
\Delta n=\frac{\pi R}{\sigma_{z} \kappa} .
$$

Since this ratio is typically large compared to 1 , the minimum required adjustment of $\kappa$ is small. Since $\mu=n / \Delta n$, the sum (A5) takes the form of an FFT at least for those $n$ which are integral multiples of $\Delta n$. Putting $n=m \Delta n$ we have

$$
\begin{gathered}
\lambda_{m \Delta n}=\frac{\kappa(-1)^{m}}{\pi} \frac{1}{J} \sum_{j=0}^{J-1} e^{-2 \pi i m j / J} \rho\left(q_{j}\right), \\
m=0,1, \ldots, \mathcal{N} ; \quad J=2 \mathcal{N} .
\end{gathered}
$$

The upper limit on $m$ comes from the Nyquist rule, which states that a mode $m$ is meaningful only if the phase $2 \pi m j / J$ changes by not more than $\pi$ from one integration point to the next (i.e., when $j$ changes by one unit).

For parameters of interest for our example $\left(\sigma_{z}=\right.$ $1 \mathrm{~cm}, R=25 \mathrm{~cm}, \kappa=6)$ the integer part of $\pi R / \sigma_{z} \kappa$ is 13 , and we can change $\kappa$ to $6.0415 \ldots$ to make $\Delta n=13$, or to $6.5449 \ldots$ to make $\Delta n=12$. It may be possible to get by with values of $n$ only in steps of $\Delta n$, using an interpolation technique to fill in the missing $n$ in the sum (20). We note, however, that a smaller $\Delta n$ can be achieved at the expense of a longer FFT. This will allow a check on accuracy of the interpolation method. Define a new FFT data vector padded with zeros:

$$
\tilde{\rho}_{j}= \begin{cases}\rho\left(q_{j}\right), & j=0,1, \ldots, J-1, \\ 0, & j=J, J+1, \ldots, 2 J-1 .\end{cases}
$$

To illustrate, suppose that the original $\Delta n$ is even. Then with $n=m \Delta n / 2$ the trapezoidal integration (A5), respecting the Nyquist rule, can be written as 


$$
\begin{aligned}
\lambda_{m \Delta n / 2} & =\frac{\kappa i^{m}}{\pi} \frac{1}{2 J} \sum_{j=0}^{2 J-1} e^{-2 \pi i m j /(2 J)} \tilde{\rho}_{j}, \\
m & =0,1, \ldots, J, \quad J=2 \mathcal{N} .
\end{aligned}
$$

By doing an FFT of twice the length in comparison to (A7), we cover the same range of $n$, but in steps half as big.

Now consider evaluation of the collective force through (20). Our method to compute $Z(n)$ in terms of Bessel functions is covered in [22]. To calculate the sum in (20) we first need a guess for the maximum required $n$. It seems that $n$ should go well beyond the point at which $Z(n) / n$ is maximum, since according to the doctrine of linear coasting beam theory modes near the maximum are likely to become unstable at high current. The maximum is around $n=600$ in our example. With $\Delta n=13$ and 400 mesh points in $q$ space $(\mathcal{N}=200)$, we can reach $n=\mathcal{N} \Delta n=$ 2600 . At first this seemed an adequate choice, and appealing because at $\mathcal{N}=200$ one can run for hundreds of synchrotron periods in modest computer time. We discovered, however, that finer meshes $(\mathcal{N}=400$ or 600 with the same $\kappa \approx 6$ ) gave slightly lower current thresholds for instability. We conclude that one should experiment, trying to see some sort of convergence of the current threshold as the mesh is refined.

Regarding the missing $n$ in the above scheme with $\Delta n>$ 1 , we note $Z(n)$ for the parallel plate model is well represented by interpolation of its values at points spaced by fairly large values $\Delta n$, say 10-20. Allowing the plotting program to provide interpolation, one cannot distinguish the graph using all $n$ from the one with spaced $n$. If $Z(n) \lambda_{n}$ had a similar property, then one could evaluate the sum of (20) using an interpolative scheme. It is certainly not clear that the interpolation will be as accurate as that of $Z(n)$ alone, but we can check the result by reducing $\Delta n$ through a longer FFT.

We consider interpolative schemes for evaluation of a general sum

$$
\begin{aligned}
S(\theta)= & \sum_{m=m_{0} \Delta n}^{\mathcal{N} \Delta n} e^{i n \theta} f(n)=e^{i n \Delta n \theta} f(\mathcal{N} \Delta n) \\
& +\sum_{m=m_{0}}^{\mathcal{N}-1} \sum_{k=0}^{\Delta n-1} e^{i(m \Delta n+k) \theta} f(m \Delta n+k) .
\end{aligned}
$$

The idea is to write $f(m \Delta n+k)$ as a low order polynomial in $k$ for $k \in[0, \Delta n-1]$, the polynomial obtained by interpolation of values $f\left(m^{\prime} \Delta n\right)$ for $m^{\prime}$ near $m$. Then the sum on $k$ can be carried out analytically, in terms of sums of geometric series and their derivatives with respect to $\theta$. In the case of quadratic interpolation this is the discrete analog of Filon's method for evaluation of Fourier transforms [37]. We state the result for linear interpolation,

$$
f(m \Delta n+k)=f(m \Delta n)+\frac{f((m+1) \Delta n)-f(m \Delta n)}{\Delta n} k .
$$

After a fairly long calculation one finds

$$
S(\theta)=\frac{1}{\Delta n} \frac{1-\cos \Delta n \theta}{1-\cos \theta} \sum_{m=m_{0}}^{\mathcal{N}-1} f(m \Delta n) e^{i m \Delta n \theta}+B(\theta),
$$

where the boundary term $B$, which will be zero in our application, in general has the form

$$
\begin{aligned}
B(\theta)= & e^{i n \Delta n \theta} f(\mathcal{N} \Delta n)+\left[\frac{1}{e^{i \theta}-1}+\frac{1-e^{-i \Delta n \theta}}{2 \Delta n(1-\cos \theta)}\right] \\
& \times\left[e^{i n \Delta n \theta} f(\mathcal{N} \Delta n)-e^{i m_{0} \Delta n \theta} f\left(m_{0} \Delta n\right)\right] .
\end{aligned}
$$

We can now compute the collective force with the help of (A12). Put $f(n)=Z(n) \lambda_{n}(t)$ and note that negative $n$ can be eliminated because $f(n)=f(-n)^{*}$. We can assume that $f(\mathcal{N} \Delta n)$ is negligible, and since $Z(0)=0$ the boundary terms drop out: $f(0)=f(\mathcal{N} \Delta n)=0$. Suppressing the time variable, we have from (20), (A2), (A12), and (A6) that

$$
\begin{aligned}
F\left(q_{j}\right) & =-2 \omega_{0} \operatorname{Re} \sum_{n=0}^{\mathcal{N} \Delta n} \exp \left(i n q_{j} \sigma_{z} / R\right) f(n) \\
& =a(j) \operatorname{Re} \sum_{m=0}^{\mathcal{N}}(-1)^{m} e^{\pi i m j / \mathcal{N}} f(m \Delta n) \\
& =a(j) \operatorname{Re} \sum_{m=0}^{2 \mathcal{N}-1} e^{2 \pi i m j / 2 \mathcal{N}} f_{m}
\end{aligned}
$$

where

$$
a(j)=-\frac{2 \omega_{0}}{\Delta n} \frac{1-\cos \left(q_{j} \pi / \kappa\right)}{1-\cos \left(q_{j} \sigma_{z} / R\right)},
$$

and

$$
f_{m}= \begin{cases}(-1)^{m} f(m \Delta n), & m=0, \ldots, \mathcal{N} \\ 0, & m=\mathcal{N}+1, \ldots, 2 \mathcal{N}-1 .\end{cases}
$$

By rewriting (A14) as (A15), we get the result as an FFT of length $2 \mathcal{N}$, providing all required $j=0,1, \ldots, 2 \mathcal{N}$. The direct Fourier transform with gaps in the $n$ spectrum, as given by (A7), meshes nicely with the inverse transform in the form (A15), obtained by interpolation.

\section{APPENDIX B: INTEGRATION METHOD FOR THE VLASOV EQUATION}

A stable method for time-domain integration of the nonlinear Vlasov equation is based on discretizing the 
Perron-Frobenius operator for the single-particle map, the latter being approximated by freezing the coherent force over sufficiently small time steps. Let $M_{\tau \rightarrow \tau+\Delta \tau}(z), z=$ $(q, p)$ be the volume preserving map describing particle trajectories (characteristics) over the time interval $[\tau, \tau+$ $\Delta \tau]$. Then the PF operator $\mathcal{M}$ associated with $M$ gives the time evolution of the distribution function:

$$
f(z, \tau+\Delta \tau)=\mathcal{M} f(z, \tau)=f\left(M^{-1}(z), \tau\right) .
$$

This is just another way of stating that the probability of finding a particle in a phase space volume element $d z$ is preserved:

$$
f(M(z), \tau+\Delta \tau) d(M(z))=f(z, \tau) d z .
$$

A discretization of $\mathcal{M}$ simply consists of choosing a finitedimensional approximation of $f$. For instance, $f$ might be described by its values on a grid $\left\{z_{i}\right\}$, with polynomial interpolation to off-grid points. In that case, evaluation of $\mathcal{M} f\left(z_{i}, \tau\right)$ would be done by interpolation, since $M^{-1}\left(z_{i}\right)$ is an off-grid point in general. In the literature the discretized PF method is often called the semi-Lagrangian method [38], but a more descriptive name would be the method of local characteristics, since it is just the traditional method of characteristics extended to self-consistent dynamics through an approximation of characteristics that is valid only locally in time.

Several different ways to approximate $M$ for small $\Delta \tau$ have been proposed, as well as different ways to represent $f$. In the seminal work of Cheng and Knorr [39], the local map $M$ was composed of three steps in a "leap frog" scheme: a drift in $q$ for time step $\Delta \tau / 2$, an increment in $p$ for time step $\Delta \tau$ accounting for both the linear motion and the coherent force, and another drift for $\Delta \tau / 2$. The PF operator for each step was discretized separately, by means of splines or Fourier series. Two of the present authors [19] took $M$ to be a rotation in phase space followed by a kick in $p$ from the coherent force, both for step $\Delta \tau$. They used locally quadratic interpolation on a grid to represent $f$, doing a single two-dimensional interpolation per time step.

In the present work we apply a method of Yabe et al. [40], which uses the Cheng-Knorr representation of $M$ but a different representation of $f$ based on the idea of cubic Hermite interpolation. In the Hermite scheme a function is represented locally as a cubic polynomial determined by the values of the function and its derivative on two adjacent mesh points. This gives an error that is $\mathcal{O}\left(h^{4}\right)$ for mesh step $h$, provided that the function has a continuous fourth derivative [41]. Yabe's scheme works with easily computed approximations to the partial derivatives along phase space axes, and requires storage of the approximated derivatives as well as function values. Since some of the derivatives are approximated crudely, one does not expect the full accuracy of Hermite interpolation. The scheme appears to work better than the method of [19] for a given mesh step, but it requires much more storage and computation time per time step. We have not yet made a careful comparison of overall efficiencies accounting for both storage and time.

The Cheng-Knorr representation of the PF operator for Eq. (13) may be stated as follows :

$$
\begin{gathered}
f^{*}(q, p, \tau)=f(q-p \Delta \tau / 2, p, \tau), \\
f^{* *}(q, p, \tau)=f^{*}\left(q, p+\left(q-I_{c} F\left(q, f^{*}\right)\right) \Delta \tau, \tau\right), \\
f(q, p, \tau+\Delta \tau)=f^{* *}(q-p \Delta \tau / 2, p, \tau) .
\end{gathered}
$$

In the Yabe scheme the function $f$ is represented by its values on a Cartesian grid, $f_{i j}(\tau)=f\left(q_{i}, p_{j}, \tau\right)$, with approximated Hermite interpolation for off-grid points. The true cubic Hermite interpolation of function values $g(x), g\left(x_{+}\right)$which fits given values of derivatives $g^{\prime}(x), g^{\prime}\left(x_{+}\right)$, where $x_{+}=x+h$, is

$$
\begin{aligned}
g(x+\xi) & \approx g(x)+g^{\prime}(x) \xi+c_{2} \xi^{2}+c_{3} \xi^{3}, \\
c_{2} & =-\frac{1}{h}\left(2 g^{\prime}(x)+g^{\prime}\left(x_{+}\right)\right)-\frac{3}{h^{2}}\left(g(x)-g\left(x_{+}\right)\right), \\
c_{3} & =\frac{1}{h^{2}}\left(g^{\prime}(x)+g^{\prime}\left(x_{+}\right)\right)+\frac{2}{h^{3}}\left(g(x)-g\left(x_{+}\right)\right) .
\end{aligned}
$$

Given $f_{i j}, \partial_{q} f_{i j}, \partial_{p} f_{i j}$ we wish to determine $f_{i j}^{*}, \partial_{q} f_{i j}^{*}, \partial_{p} f_{i j}^{*}$ through (B3). First, $f_{i j}^{*}$ is determined by Hermite interpolation of $f$ with respect to its first argument, then $\partial_{q} f_{i j}^{*}$ is given by the derivative of that interpolation evaluated at grid point $(i, j)$. The other derivative $\partial_{p} f_{i j}^{*}$ is approximated more roughly, as follows. For small $\Delta \tau$ we have

$$
\begin{aligned}
\partial_{q} f(q, p, \tau) & \approx \frac{f(q, p, \tau)-f(q-p \Delta \tau / 2, p, \tau)}{p \Delta \tau / 2} \\
& =\frac{f^{*}(q+p \Delta \tau / 2, \tau)-f^{*}(q, p, \tau)}{p \Delta \tau / 2} \\
& \approx \partial_{q} f^{*}(q, p, \tau),
\end{aligned}
$$

hence

$$
\begin{gathered}
\frac{1}{2}\left(\partial_{q} f(q, p, \tau)+\partial_{q} f^{*}(q, p, \tau)\right) \\
\quad \approx \frac{f(q, p, \tau)-f^{*}(q, p, \tau)}{p \Delta \tau / 2} .
\end{gathered}
$$

Multiplying (B8) by $p \Delta \tau / 2$ and differentiating with respect to $p$, we find

$$
\begin{aligned}
\partial_{p} f^{*}(q, p, \tau) \approx & \partial_{p} f(q, p, \tau) \\
& -(\Delta \tau / 4) \partial_{p}\left(p \partial_{q}\left(f+f^{*}\right)(q, p, \tau)\right) .
\end{aligned}
$$

Finally, we approximate $\partial_{p}$ in the last term of (B9) by central divided differences on the grid with cell size $\Delta p$ in the $p$ direction. This gives the desired algorithmic value 


$$
\begin{aligned}
\partial_{p} f_{i j}^{*}= & \partial_{p} f_{i j}-\frac{\Delta \tau}{4} \frac{1}{2 \Delta p}\left[p_{j+1}\left(\partial_{q} f_{i, j+1}+\partial_{q} f_{i, j+1}^{*}\right)\right. \\
& \left.-p_{j-1}\left(\partial_{q} f_{i, j-1}+\partial_{q} f_{i, j-1}^{*}\right)\right] .
\end{aligned}
$$

One can now treat (B4) and (B5) in a similar way, finally finding $f_{i j}(\tau+\Delta \tau), \partial_{q} f_{i j}(\tau+\Delta \tau), \partial_{p} f_{i j}(\tau+\Delta \tau)$, which are starting values for the next time step. One could have used $f$ rather than $\left(f+f^{*}\right) / 2$ in the last term of (B9), but the latter is alleged to enhance stability of the algorithm. A noteworthy feature of this scheme is that the quality of the interpolation in phase space depends on smallness of $\Delta \tau$, because of (B7), whereas there is no such dependence in the methods of $[19,39]$.

[1] J. Schwinger, "On Radiation by Electrons in a Betatron," unpublished report, 1945, transcribed in Lawrence Berkeley National Laboratory Report No. LBL-39088, 1996.

[2] J. Schwinger, Phys. Rev. 75, 1912 (1949).

[3] L. Schiff, Rev. Sci. Instrum. 17, 6 (1946).

[4] J. Nodvick and D. Saxon, Phys. Rev. 96, 180 (1954).

[5] R. Warnock and P. Morton, Part. Accel. 25, 113 (1990).

[6] K.-Y. Ng, Part. Accel. 25, 153 (1990).

[7] T. Nakazato et al., Phys. Rev. Lett. 63, 1245 (1989).

[8] G. Carr, S. Kramer, J. Murphy, R. Lobo, and D. Tanner, Nucl. Instrum. Methods Phys. Res., Sect. A 463, 387 (2001).

[9] J. M. Byrd et al., in Proceedings of the European Particle Accelerator Conference, Paris, 2002 (EPS-IGA and CERN, Geneva, 2002), p. 659.

[10] J. M. Byrd et al., Phys. Rev. Lett. 89, 224801 (2002).

[11] U. Arp et al., Phys. Rev. ST Accel. Beams 4, 054401 (2001).

[12] Å. Andersson, M. Johnson, and B. Nelander, Opt. Eng. 39, 3099 (2000).

[13] M. Venturini and R. Warnock, Phys. Rev. Lett. 89, 224802 (2002).

[14] M. Abo-Bakr, J. Feikes, H. Holldack, G. Wüstefeld, and H.-W. Hübers, Phys. Rev. Lett. 88, 254801 (2002);90, 094801 (2003).

[15] P. Podobedov et al., in Proceedings of the IEEE Particle Accelerator Conference, Chicago, IL, 2001 (IEEE, Piscataway, NJ, 2002), p. 1921; S. Kramer et al., in Proceedings of the European Particle Accelerator Conference, Paris, 2002 (Ref. [9]), p. 1523.

[16] F. Sannibale, J. M. Byrd, Á. Loftsdóttir, M. Venturini, M. Abo-Bakr, J. Feikes, K. Holldack, P. Kuske, G. Wüstefeld, H.-W. Hübers, and R. Warnock, Phys. Rev. Lett. 93, 094801 (2004).

[17] R. J. Loewen, Ph.D. thesis, Stanford University [Stanford Linear Accelerator Center Report No. SLAC-R-632, 2003].
[18] W. C. Barry et al., in Proceedings of the European Particle Accelerator Conference, Paris, 2002 (Ref. [9]), p. 656.

[19] R. Warnock and J. Ellison, in Proceedings of the 2nd ICFA Advanced Workshop on Physics of High Brightness Beams, Los Angeles, 1999 (World Scientific, Singapore, 2000); Report No. SLAC-PUB-8404, 2000.

[20] S. Heifets, G. Stupakov, and S. Krinsky, Phys. Rev. ST Accel. Beams 5, 064401 (2002).

[21] Zh. Huang and K.-J. Kim, Phys. Rev. ST Accel. Beams 5, 074401 (2002).

[22] R. Warnock, R. D. Ruth, M. Venturini, and J. A. Ellison, Phys. Rev. ST Accel. Beams 8, 014402 (2005).

[23] Zh. Huang and R. D. Ruth, Phys. Rev. Lett. 80, 976 (1998).

[24] M. Venturini, in Proceedings of the IEEE Particle Accelerator Conference, Chicago, IL, 2001 (Ref. [15]), p. 2961.

[25] S.Y. Lee, Accelerator Physics (World Scientific, Singapore, 2000), Chap. 3.

[26] R. Warnock and M. Venturini, in Proceedings of the Particle Accelerator Conference, Portland, OR, 2003 (IEEE, Piscataway, NJ, 2003), paper RPPB061.

[27] J. B. Murphy, S. Krinsky, and R. L. Gluckstern, Part. Accel. 57, 9 (1997)

[28] D. Boussard, CERN Report No. CERN LABII/RF/INT/ 75-2, 1975 .

[29] J. M. Wang and C. Pellegrini, in Proceedings of the 11th International Conference on High Energy Accelerators, Geneva, 1980 (Birkhauser-Verlag, Basel, 1980), p. 554.

[30] S. Krinsky and J. M. Wang, Part. Accel. 17, 109 (1985).

[31] G. R. Bart and R. L. Warnock, SIAM J. Math. Anal. 4, 609 (1973).

[32] G. R. Bart, J. Math. Anal. Appl. 79, 48 (1981).

[33] R. Warnock, G. Stupakov, M. Venturini, and J. A. Ellison, in Proceedings of the European Particle Accelerator Conference, Lucerne, 2004, http://accelconf.web.cern.ch/ accelconf/e04/default.htm, paper WEPLT159.

[34] Handbook of Mathematical Functions, edited by M. Abramowitz and I. A. Stegun, Applied Mathematics Series Vol. 55 (National Bureau of Standards, Washington, DC, 1972).

[35] R. W. Hockney and J. W. Eastwood, Computer Simulation Using Particles (Institute of Physics, Bristol, 1988).

[36] R.C. Davidson and H. Qin, Physics of Intense Particle Beams (World Scientific, Singapore, 2001), Sec. 8.5.

[37] L. N. G. Filon, Proc. R. Soc. Edinburgh A 49, 38 (1928).

[38] F. Filbet and E. Sonnendrücker, Comput. Phys. Commun. 150, 247 (2003).

[39] C. Z. Cheng and G. Knorr, J. Comput. Phys. 22, 330 (1976).

[40] T. Nakamura and T. Yabe, Comput. Phys. Commun. 120, 122 (1999), and earlier work cited therein.

[41] C. de Boor, A Practical Guide to Splines (Springer, New York, 2001), revised edition, p. 40. 